\title{
L'ONDA «G » DI GUTENBERG NEL TERREMOTO DELLE AZZORRE DEL 25 NOVEMBRE 1941
}

\section{Domenico Di Filippo}

L'onda " $G$ », osservata per la prima volta da Gutenberg e Richter nel forte terremoto delle isole Salomone del 3 ottobre 1931, è un'onda superficiale a lunghissimo periodo ed a carattere nettamente trasversale. Essa viene registrata solo in caso di terremoti di notevole intensità e bene solo da sismografi a lungo periodo proprio.

Esempi particolarmente chiari ed interessanti di questo tipo di onda si sono ottenuti in occasione del fortissimo terremoto delle Azzorre del 25 novembre 1941. Data la notevole intensità all'origine, l'energia è stata sufficiente a determinare in parecchi osservatori registrazioni oltre che dell'onda diretta $G_{1}$, anche della $G_{\text {, , }}$ cioè l'onda che ha percorso l'arco più lungo del cerchio massimo epicentro-stazione, ed inoltre della $G_{3}$, giunta alla stazione dopo aver compiuto, passando per l'epicentro, l'intero giro della Terra. Nei sismogrammi degli Osservatori sismici di Roma I.N.G. e di Rio de Janeiro è stato inoltre possibile individuare le $G_{t}$ e $G_{5}$.

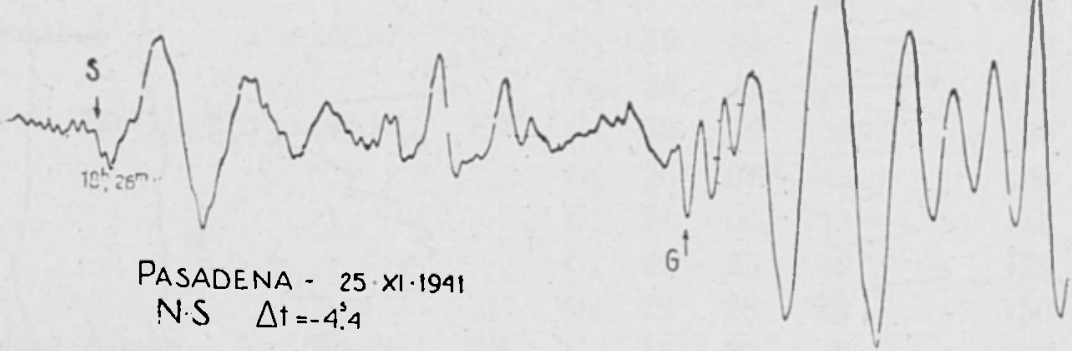

Fig. 1

Poiché queste onde raramente si presentano in condizioni migliori, ho pensato di farne oggetto di uno studio particolare. In una nota precedente ho calcolato, con i dati di 36 stazioni, le seguenti coordinate epicentrali e l'ora origine, riferita al T.M.G.

$$
\begin{array}{ll}
\varphi_{\mathrm{o}}=37^{\circ} & 25^{\prime}, 41-5^{\prime}, 2 \mathrm{~N} \\
\lambda_{\mathrm{o}}=19^{\circ} & 00^{\prime}, 65-3^{\prime}, 0 \mathrm{~W} \\
H_{\mathrm{o}}=18^{\mathrm{h}} & 03^{\mathrm{m}} 54^{\circ}, 7-0^{\mathrm{s}}, 41
\end{array}
$$



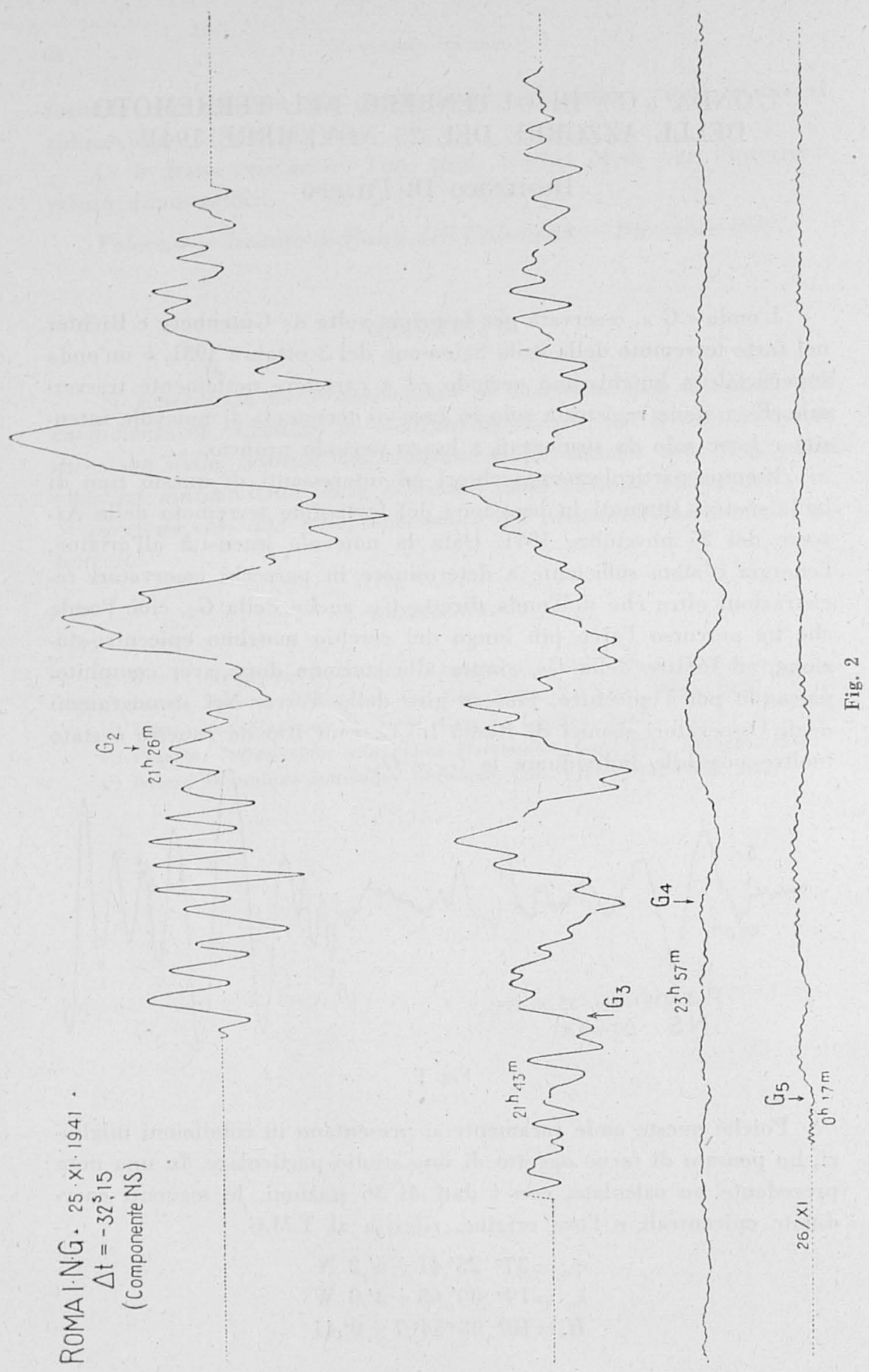
Nella tabella seguente sono elencati gli osservatori, con le distanze epicentrali, le varie fasi della " $\mathrm{G}$ " individuate, con $\mathrm{i}$ corrispondenti tempi di arrivo e periodi:

\begin{tabular}{|c|c|c|c|c|c|c|c|}
\hline Lisbona & $\Delta=7^{\circ}, 88$ & NS & $G$. & $20^{\mathrm{h}}$ & $30^{\mathrm{m}}$ & $39 * 3$ & $T=123^{\mathrm{s}}$ \\
\hline Averroes & $\Delta=10^{\circ}, 32$ & EW & $G_{\text {n }}$ & 20 & 31 & 11 & 90 \\
\hline \multirow[t]{5}{*}{ Roma } & $\Delta=25^{\circ}, 00$ & NS & $G_{1}$ & 18 & 13 & 58 & 40 \\
\hline & & & $G$ & 20 & 25 & 48 & 110 \\
\hline & & & $G_{3}$ & & 43 & 20 & 125 \\
\hline & & & $G_{4}$ & 22 & 57 & 53 & 150 \\
\hline & & & $G_{\overline{5}}$ & 23 & 16 & 40 & 160 \\
\hline Jena & $\Delta-=25^{\circ}, 55$ & NS & $G_{1}$ & 18 & 14 & 23,6 & 50 \\
\hline Praga & $\Delta=27^{\circ}, 02$ & $\mathrm{NS}$ & $G_{1}$ & 18 & 15 & 02,5 & 50 \\
\hline \multirow[t]{2}{*}{ Uppsala } & $\Delta=32^{\circ}, 35$ & $\mathrm{FW}$ & $G_{\text {, }}$ & 20 & 20 & 17,4 & 140 \\
\hline & & & $G_{3}$ & & 45 & 38 & 140 \\
\hline \multirow[t]{3}{*}{ Sofia } & $\Delta=32^{\prime \prime}, 58$ & NS & $G_{1}$ & 18 & 17 & 32,3 & 50 \\
\hline & & & $G_{2}$ & 20 & 20 & 32,3 & 110 \\
\hline & & & $G_{3}$ & & 49 & 26,3 & 120 \\
\hline Ottawa & $\Delta=42^{\circ}, 49$ & NS & $G_{1}$ & 18 & 21 & 24 & 60 \\
\hline \multirow[t]{3}{*}{$\mathrm{K}_{\text {sara }}$} & $\Delta=44^{n}, 24$ & NS & $G_{1}$ & 18 & 22 & 10 & 44 \\
\hline & & & $G_{\text {. }}$ & 20 & 13 & 40,4 & 108 \\
\hline & & & $G_{3}$ & & 53 & 00 & 132 \\
\hline \multirow[t]{2}{*}{ San Juan } & $\Delta=45^{\circ}, 23$ & EW & G., & 20 & 16 & 00,8 & 110 \\
\hline & & & $G_{3}$ & & 53 & 04 & 130 \\
\hline \multirow[t]{2}{*}{ Columbia } & $\Delta=49^{\circ}, 67$ & NS & $G_{1}$ & 18 & 24 & 20 & 48 \\
\hline & & & G., & 20 & 13 & 32 & 110 \\
\hline \multirow[t]{5}{*}{ Rio de Janeiro } & \lrcorner$=64^{\circ}, 18$ & EW & $G_{1}$ & 18 & 30 & 12 & 60 \\
\hline & & & G., & 20 & 07 & 45 & 100 \\
\hline & & & $G_{3}$ & 21 & 00 & 11 & 120 \\
\hline & & & $G_{4}$ & 22 & 35 & 45 & 125 \\
\hline & & & $G_{5}$ & 23 & 28 & 42 & 150 \\
\hline \multirow[t]{3}{*}{ College Alaska } & $\Delta=70^{\circ}, 49$ & EW & $G_{1}$ & 18 & 32 & 40 & 60 \\
\hline & & & G., & 20 & 04 & 08 & 100 \\
\hline & & & $G_{3}$ & 21 & 02 & 20 & 120 \\
\hline Pasadena & $\Delta-76^{\circ}, 53$ & NS & $G_{1}$ & 18 & 35 & 00 & 82 \\
\hline La Plata & $\Delta=80^{\circ}, 54$ & FW & $G_{1}$ & 18 & 37 & 38 & 48 \\
\hline
\end{tabular}

A Lisbona, Averroes, Uppsala e San Juan, non è stato possibile individuare la « $G$ » al primo passaggio, perché mascherata da altri tipi d'onda a più corto periodo. $A$ Roma invece i fotosismografi sen- 


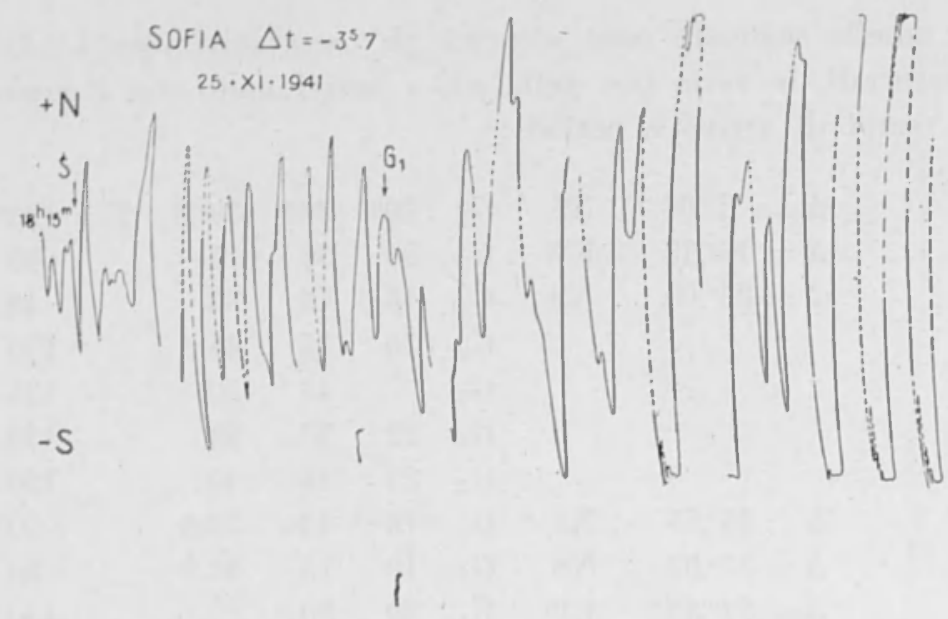

$20^{h} 19^{m}$ $I_{G_{2}}$

$20^{h_{48} m} \quad G_{3}$

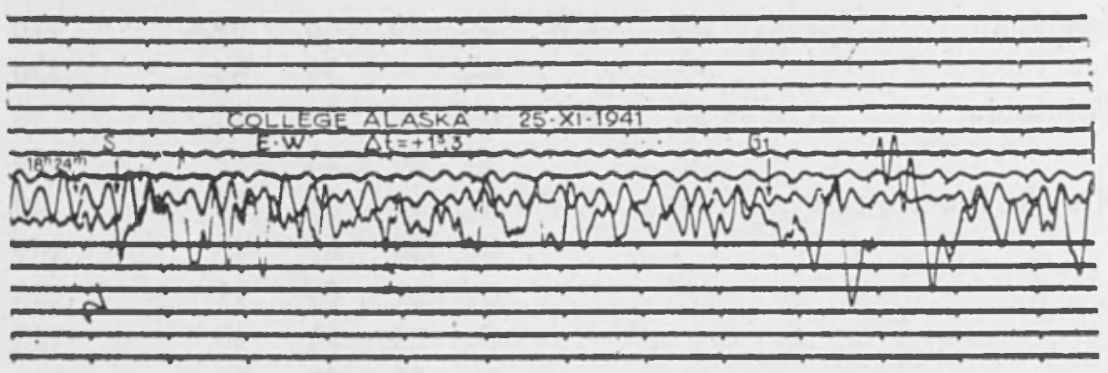

AVERROES $\triangle t=+7^{3}, \quad 25 \cdot \times 1 \cdot 1241$

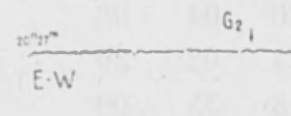

LISBONA $\quad \Delta t=-3447: 7 \quad 25 \cdot x_{1} \cdot 1941$

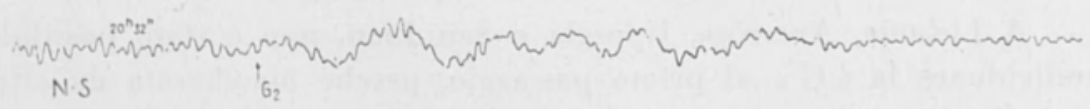

Fig. 3 
sibili, a lungo periodo, non l'hanno registrata a causa della grande ampiezza. Il valore di questa ampiezza, calcolato da Caloi, ̀̀ risultato di $30 \mathrm{~cm}$ circa dalla linea di riposo. Le $G_{i,}, G_{3,}, G_{4}$ e $G_{5}$ hanno invece determinato, sulla componente $N S$, registrazioni molto nette (fig. 2 ).

Si sono ottenuti esempi chiari a Sofia, Averröes, Lisbona, e College Alaska (fig. 3 । e particolarmente interessante è risultata la registrazione della $G_{1}$ ottenuta a Pasadena (fig. 1) con un fotosismografo speciale a lunghissimo periodo proprio $\left(T=70^{\mathrm{s}}\right)$.

Anche in questo terremoto si è rilevata la natura nettamente trasversale-tangenziale di quest'onda in quanto le registrazioni delle stazioni poste quasi ad Est dell'epicentro hanno dato esempi notevoli solo nella componente NS, Roma, Ksara, Sofia, Lisbona, mentre a Rio de Janeiro l'onda è stata registrata solo nella componente $E[F$.

Dromocrona. - Con i tempi di tragitto dell'onda $G_{1}$, relativi alle stazioni di Roma, Jena, Praga, Sofia, Ottawa, Ksara, Columbia, Rio de Janeiro, College Alaska, Pasadena, La Plata, ho calcolato, col metodo dei minimi quadrati, per la dromocrona, la equazione seguente:

$$
t^{*}=\frac{\Delta^{\circ}}{0.04051 \div 0.00039}-0^{*}, 026+0^{*}, 513
$$

con una velocità apparente di $4,50 \mathrm{~km} / \mathrm{s}$ (e l'inizio coincidente con il tempo origine del terremoto).

Come verifica del calcolo lo schema $[l l .2]=3.743$ e $[v v]=3.744$ sono risultati praticamente uguali, come vuole la teoria degli errori.

Nello specchietto seguente riporto i valori delle velocità medie apparenti delle varie " $G$ " per $i$ singoli osservatori e $i$ valori medi trovati da Gutenberg e Ricbter per il citato terremoto.

La velocità trovata da Gutenberg e Ricbter per il terremoto delle isole Salomone è risultata variabile tra 4,39 e $4,63 \mathrm{~km} / \mathrm{s}$, per distanze da 5.600 a $16.000 \mathrm{~km}$; in questo caso i limiti per la velocità risultano pressoché uguali e cioè 4,38 e $4,58 \mathrm{~km} / \mathrm{s}$ mentre il limite inferiore per la distanza epicentrale di osservazione risulta notevolmente ridotto con i $900 \mathrm{~km}$ circa della stazione di Lishona.

Confrontando i risultati si vede che per la $G_{1}$ il valore trovato di $4,50 \mathrm{~km} / \mathrm{s}$ è in ottimo accordo con $4,487 \mathrm{~km} / \mathrm{s}$ di Gutenberg e Ricbter e per le altre due coppie di valori relativi alla $G_{\text {g }}$ ed alla $G_{: b}$ l'accordo è soddisfacente, considerato che le differenze risultano minori di $0,02 \mathrm{~km} / \mathrm{s}$, più che giustificabili con gli errori di osservazione. 


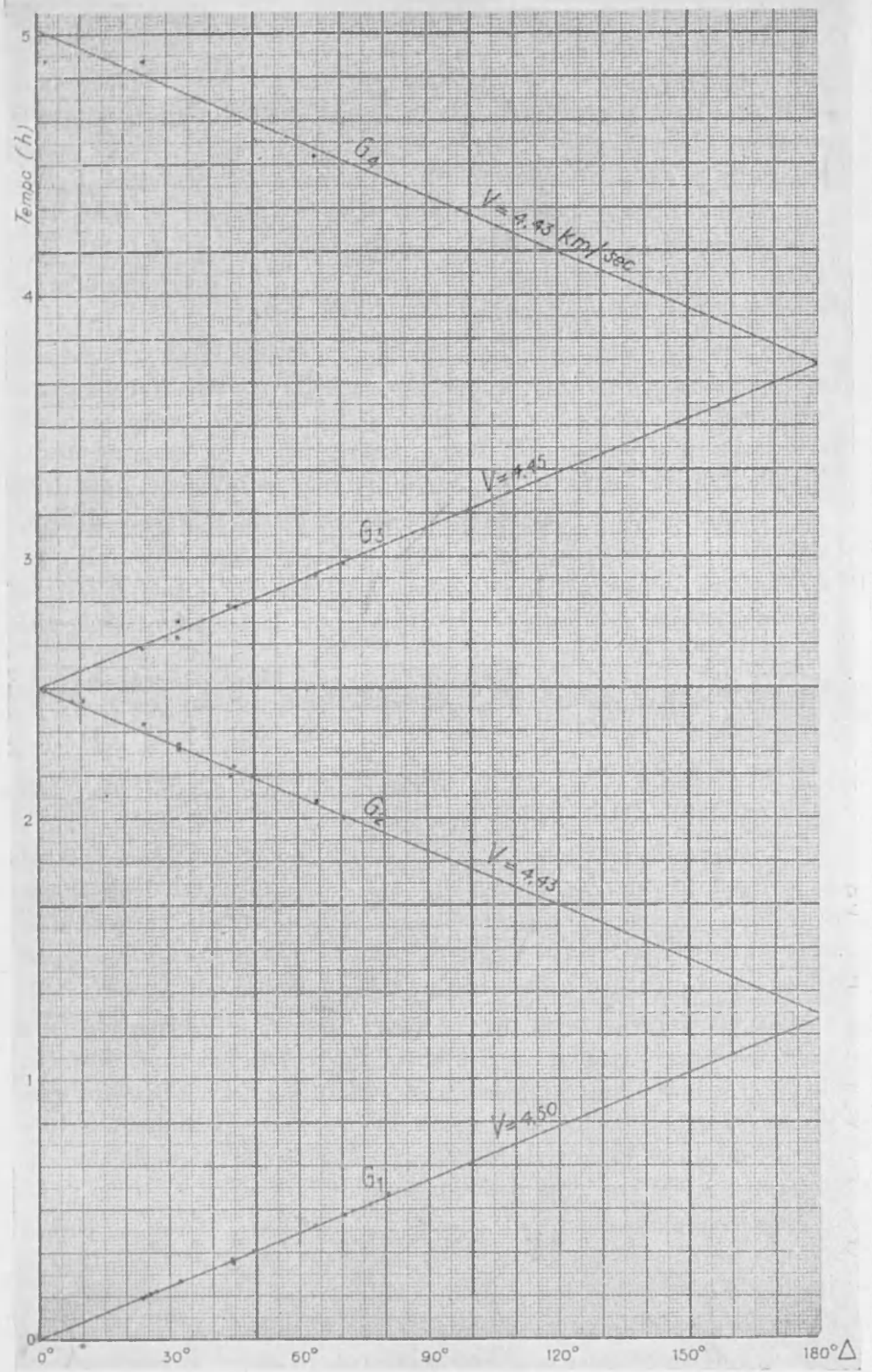

Fig. 4 - Dromocrona della « $\mathrm{G}$ » . Terremoto del 25 novembre 1941 


\begin{tabular}{|c|c|c|c|c|c|}
\hline \multirow{2}{*}{ Stazioni } & \multicolumn{5}{|c|}{ V'elocità $\mathrm{km} / \mathrm{s}$} \\
\hline & $\mathbf{G}_{1}$ & $\mathrm{G}_{2}$ & $G_{3}$ & $G_{i}$ & $G_{5}$ \\
\hline $\begin{array}{l}\text { Lisbona } \\
\text { Averroes } \\
\text { Roma } \\
\text { Jena } \\
\text { Praga } \\
\text { Uppsala } \\
\text { Sofia } \\
\text { Ottawa } \\
\text { Ksara } \\
\text { Sant Juan } \\
\text { Columbia } \\
\text { Rio de Janeiro } \\
\text { College Alaska } \\
\text { Pasadena } \\
\text { La Plata }\end{array}$ & $\begin{array}{l}- \\
\overline{4.52} \\
451 \\
450 \\
\overline{4} \\
4.50 \\
449 \\
\overline{4.50} \\
452 \\
4.53 \\
4.58 \\
4.42\end{array}$ & $\begin{array}{l}443 \\
4.40 \\
438 \\
\\
4 \overrightarrow{45} \\
4.44 \\
4 \overline{41} \\
4.41 \\
4.43 \\
4.42 \\
4.46 \\
- \\
-\end{array}$ & $\begin{array}{l}\overline{-} \\
\overline{4.47} \\
\overline{4} \\
4.49 \\
49 \\
4.43 \\
4.44 \\
4.46 \\
4.47 \\
-\end{array}$ & $\begin{array}{l}- \\
4.38 \\
- \\
- \\
- \\
- \\
- \\
44 \\
- \\
-\end{array}$ & $\begin{array}{l}- \\
441 \\
\overline{-} \\
\overline{-} \\
\overline{-} \\
\overline{-} \\
4.4 \\
\overline{-} \\
-\end{array}$ \\
\hline & 4.500 & 4.433 & 4.450 & 443 & 444 \\
\hline Gutenberg e Richter & 4.487 & 4.460 & 4.430 & - & - \\
\hline
\end{tabular}

Roma - Istituto Nazionale di Geofisica - Dicembre 1949.

\section{RIASSUNTO}

Si espongono $i$ risultati di uno studio sull'onda « $G$ » di Gutenberg, registrata nel terremoto delle Azzorre del 25 novembre 1941.

\section{BIBLIOGRAFIA}

B. Gutenderg and C. F. Richter: On seismic waves - Gerlands Beitrage zur Geo. physik. Band 43 Heft 1/2. 1934.

P. Calor: Nuova onda a lungo periodo oscillante nel piano principale; sue caratteristiche e confronto con londa " $\mathrm{G}$ ». Boll. Comitato per la Geodesia e Geofisica del C.N.R. serie II, n. 3, 1936.

P. Calo1: Onde sismiche superficiali e loro assorbimento da parte del mezzo. L'Universo, Riv, dell'Istituto Geografico Militare, n. 7, 1942.

D. Di Filippo: Il terremoto delle Azzorre del 25 novembre 1941. Annali di Geofisica, vol. II, n. 3, 1949. 